\title{
\& Research Square \\ Application of Machine Learning for Inter Turn Fault Detection in Pumping System
}

Nabanita Dutta

Vellore Institute of Technology

Palanisamy Kaliannan ( $\sim$ kpalanisamy@vit.ac.in )

Vellore Institute of Technology

Paramasivam Shanmugam

Esab India limited

\section{Research Article}

Keywords: Induction motor, Pump, Inter turn fault, ANN, ANFIS, Machine learning

Posted Date: March 8th, 2022

DOI: https://doi.org/10.21203/rs.3.rs-1370571/v1

License: (1) This work is licensed under a Creative Commons Attribution 4.0 International License.

Read Full License 


\title{
Application of machine learning for inter turn fault detection in pumping system
}

\author{
Nabanita Dutta ${ }^{1+}$, Palanisamy Kaliannan ${ }^{1+}{ }^{\star}$, Paramasivam Shanmugam $^{2,+}$ \\ ${ }^{1}$ Department of Energy and Power Electronics, School of Electrical Engineering, Vellore Institute of Technology, Vellore \\ 632014, India \\ ${ }^{2}$ Esab India limited, Chennai, India \\ *kpalanisamy@vit.ac.in \\ +these authors contributed equally to this work
}

\begin{abstract}
Pump fault diagnosis is essential for maintenance and safety of the device as it is an important appliance used in various major sectors. Fault diagnosis at proper time can reduce maintenance cost and save energy. In this article a Simulink model based on mathematical equations has been built for analyzing the effects of parameter estimation of three phase induction motor based centrifugal pump in inter turn fault condition. The inter turn fault causes huge increase of current which severely affects the parameters of both motor and pump and these have been analysed by simulation through Matlab Simulink model. Later, the results are verified by hardware in loop (HIL) based simulator. In this paper machine learning (ML) based artificial neural network (ANN) and ANFIS (ANN and Fuzzy) models have been applied for fault detection ANN and ANFIS based models provide a satisfactory level of accuracy. These models provide accurate training and testing results. Based on root mean square error (RMSE), R2, prediction accuracy and mean validation value these models are compared to find out which is more suitable for this experiment. Various supervised algorithms are compared with ANN, ANFIS and lastly found which is the most suitable for this experiment.
\end{abstract}

Keywords: Induction motor, Pump, Inter turn fault, ANN, ANFIS, Machine learning

\section{Introduction}

The induction motor is commonly used device which is indispensable for various industries and have received augmented attention for their robust construction, high performance, reliability and maintenance cost [1]. Any kind of fault in induction motor causes drastic consequences in the devices connected with the motor and entire system. If pump is connected with faulty induction motor the head value will change, flow rate will change and huge vibration will create severe damage [2]. Breakdown of entire system not only causes system damage but also creates huge energy loss and, unplanned sudden downtime causes and huge maintenance cost. It is reported that $30 \%$ to $40 \%$ failure is seen in induction motor for stator inter turn fault [3]. This is actually electrical fault and this electrical fault is very sensitive which causes severe damage. Only $10 \%$ to $20 \%$ inter turn fault causes huge rising of current in induction motor which causes insulation losses in the windings [4]. Electrical faults are categorized both as stator and rotor fault [5]. The rotor fault which is seen in induction motor is broken rotor bar. Stator faults are mainly three faults like phase to phase fault, inter turn fault and phase to ground fault. Among those inter turn fault is major and critical fault [6]. This inter turn fault not only hampers induction motor operation but also pumping operation. Besides mechanical and hydraulic fault, electrical fault also hampers the pump performance. The centrifugal pump is rotating machine used to transfer fluid through pipes. Sudden shutdown of pumping system causes huge loss for maintenance [7]. It has been analysed that $70 \%$ of the maintenance cost is seen for the pumping system. So it is required to improve the maintenance technology for reducing cost. Various researches have been done for inter turn fault detection in induction motor. Voltage between lines, neutral and star point of motor were used for fault detection. This was used as model of the motor and an imbalance was created due to inter turn short circuit fault. Before the total breakdown and major damage of the devices this imbalance should be identified. [8]. Negative sequence impedance was estimated and used as fault indicator in a research. The negative sequence impendence was seen due to imbalance in the motor. Oscillation used for Park transformation current was used for fault detection which were created for imbalance. To identify this problem space vector analysis is required [9]. Electrical fault can be detected by motor current signature analysis (MCSA) and vibration analysis. For the estimation of negative impedance in the motor, robustness with respect to unbalanced voltage supply was added as an approach [10]. Frequency spectrum and fast Fourier transformation (FFT) analysis also are helpful for fault detection in induction motor. Wavelet package transformations (WPT) and FFT were used along with some sort of classifier in some work [11] [12]. About faults in the stator for extracting knowledge higher order statistics (HOS) was used. For rotating machine, pump health monitoring is possible for analyzing supply voltage, current, and power, torque and speed value. Faults in the machine can be analysed and 
identified by harmonic analysis also. In this case stator current produces instantaneous harmonic current [13]. Parameter estimation technique also performs the pump health monitoring process. Harmonic frequencies can analyse machine health. In hazardous and extreme environmental conditions where access of the machine is difficult such as nuclear power plant, , paper mill, chemical plant and on shore and off shore facilities, this method is applied for health monitoring of the machines [14] [15]. MCSA is an electric machinery monitoring technique which has wide application mainly in heavy industry. It can be used for detecting motor faults. Still, the limitation of this method is it will perform better if it is used in conjunction with other technologies like fast Fourier transformation (FFT), Fuzzy logic, and Park's vector analysis. The latest development in artificial intelligence (Al) is 'transfer learning', which can detect failure patterns of different devices. This technique can be used instead of MCSA and able to find out localized anomalies and has been suggested in place of MCSA for diagnosing bearing faults, impeller breaking faults in an induction motor[16].

This proposed article presents stator inter turn fault of three phase induction motor based pumping system and specially inter turn fault has been described here as it is a major and vital problem in most of the industrial sectors. Reducing operational and maintenance cost is the main aim of most of the Industries, which leads to increase in importance of condition monitoring of the machine. Requirement of additional equipment and maintenance cost are the burden for industries due to sudden failure of machines. Health monitoring of the winding of the machine is very important to avoid the losses as inter turn fault causes damage of the windings. In this research article overall stator winding fault has been discussed in next sections and how the parameters of both motor and pump are affected by stator fault that is also described. As continuous monitoring and prediction of fault detection are possible through $\mathrm{ML}$ based algorithms in best suited way, ML algorithm based ANN and ANFIS models are applied for detecting inter turn stator fault in induction motor based pumping system. Both of these models are used in the proposed research and performances of both algorithms have been compared. Moreover various supervised algorithms are compared with ANN and ANFIS to find out the better result.

\section{Proposed Model}

The proposed research has been done based on some mathematical equations of pumping system which is coupled with induction motor. These equations are applied for building the matlab simulink model of induction motor based pumping system. As fault creation in real time system causes major damage in the system, so mathematical equation based simulink model has been developed for the experimental purpose for analyzing the healthy and faulty situation. At first the model has been analysed in healthy condition without changing any parameter value. Then to analyse the faulty situation phase $\mathrm{A}$ winding has been sorted such that the current in phase A suddenly increases and it helps to increase the phase B and phase $\mathrm{C}$ current also. Due to changes of current value, torque and speed value also change and the pump parameters like pressure, flow rate are forced to change as pump is coupled with induction motor and runs in same speedup to three levels of subheading are permitted. Subheadings should not be numbered.

\section{Mathematical model of induction motor based pumping system Subsection}

In this section various equations have been described which express voltage, current, and flux of stator and rotor of induction motor.

$$
\begin{gathered}
V_{S}=R_{S} I_{S}+P \lambda_{S} \\
\text { Where } R_{r} I_{r}+P \lambda_{S} \\
V_{S}=\left[\begin{array}{llll}
V_{a s 1} & V_{a s 2} & V_{b s} & V_{c s}
\end{array}\right]^{T} \\
I_{S}=\left[\begin{array}{llll}
I_{a s} & \left(I_{a s}-I_{f}\right) & I_{b s} & I_{c s}
\end{array}\right]^{T} \\
I_{r}=\left[\begin{array}{llll}
I_{a r} & I_{b r} & I_{c r}
\end{array}\right]^{T} \\
\lambda_{S}=\left[\begin{array}{llll}
\lambda_{a s 1} & \lambda_{a s 2} & \lambda_{b s} & \lambda_{c s}
\end{array}\right]^{T}
\end{gathered}
$$

$V$ represents the voltage, I shows current, flux is represented as $\lambda_{i}$, here, " $s$ " and " $r$ " represent stator and rotor respectively, $a, b, c$ denote the three phase system. $a s_{1}, a s_{2}$ denote unfaulty and faulty part of stator respectively. Here $P$ is Laplace operator, derivative operator $\frac{d}{d t}$ is replaced by $P$.

$$
V_{a s 2}=\beta R_{s}\left(I_{a s}-I_{f}+\rho \lambda_{a s 2}\right)=R_{f} I_{f}
$$




$$
\begin{aligned}
& \lambda_{s}=L_{s} I_{s}+L_{s r} I_{r} \\
& \lambda_{r}=\left[L_{s r}\right]^{T} I_{s}+L_{r} I_{r}
\end{aligned}
$$

Shorted part of stator winding voltage is shown by these equations. $\beta$ denotes shorted turn.

The resistance matrix is shown as

$$
\begin{aligned}
& R_{s}=R_{s} \operatorname{diag}[(1-\beta) \\
& R_{r}=R_{r}[I]_{3 \times 3}
\end{aligned}
$$

Here mutual inductance and self-inductance of stator winding have been represented by the equations [12-14].

$$
\begin{aligned}
& L_{s}=L_{l s} \operatorname{diag}[(1-\beta) \quad \beta \quad 1 \quad 1]+\left[\begin{array}{cccc}
(1-\beta)^{2} & \beta *(1-\beta) & -\frac{(1-\beta)}{2} & -\frac{(1-\beta)}{2} \\
\beta *(1-\beta) & (\beta)^{2} & -\frac{\beta}{2} & -\frac{\beta}{2} \\
-\frac{(1-\beta)}{2} & -\frac{\beta}{2} & 1 & -\frac{1}{2} \\
-\frac{(1-\beta)}{2} & -\frac{\beta}{2} & -\frac{1}{2} & 1
\end{array}\right] \\
& L_{s r}=L_{m s}\left[\begin{array}{ccc}
(1-\beta) * \cos \left(\theta_{r}\right) & (1-\text { turn }) * \cos \left(\theta_{r}+2 \pi / 3\right) & (1-\beta) * \cos \left(\theta_{r}-2 \pi / 3\right) \\
\beta * \cos \left(\theta_{r}\right) & \beta * \cos \left(\theta_{r}+2 \pi / 3\right) & \beta * \cos \left(\theta_{r}-2 \pi / 3\right) \\
\cos \left(\theta_{r}-2 \pi / 3\right) & \cos \left(\theta_{r}\right) & \cos \left(\theta_{r}+2 \pi / 3\right) \\
\cos \left(\theta_{r}+2 \pi / 3\right) & \cos \left(\theta_{r}-2 \pi / 3\right) & \cos \left(\theta_{r}\right)
\end{array}\right] \\
& L_{r}=\left[\begin{array}{ccc}
L_{l r}+L_{R M} & -\frac{L_{R M}}{2} & -\frac{L_{R M}}{2} \\
-\frac{L_{R M}}{2} & L_{l r}+L_{R M} & -\frac{L_{R M}}{2} \\
-\frac{L_{R M}}{2} & -\frac{L_{R M}}{2} & L_{l r}+L_{R M}
\end{array}\right]
\end{aligned}
$$

$\beta$ represents number of turn in phase $a, \theta_{r}$ represents rotor position, $L_{s}$ shows self-inductance, $L_{r}$ shows rotor self-inductance and $L_{s r}$ represents stator to rotor mutual inductance. Water is pumped out from constant level water tank and the pumping system consists of water tank, asynchronous three phase induction motor and other parts. The tank receives liquid with input flow represented by $q_{v_{1}}$ Output flow of control valve is represented by $q_{v_{2}}$. With the help of fluid mechanics and fundamental laws of physics, analysis of the dynamics of plant has been done and a mathematical model is developed [17]. This mathematical model includes the mathematical models of centrifugal pump and tank. The counterpart of Newton's law of force is that angular acceleration is proportional to the torque on the axis. So, the equations show the motion for the motor and pump set.

$$
J \frac{d \omega}{d t}=M_{a}-M_{p}=M_{M T}-\left(M_{p}+M_{\zeta}\right)
$$

$J$ shows the moment of inertia. Here moment of inertia is constant of proportionality in specific case. Active torque of asynchronous motor is shown by $M_{M T}$ and accleration torque is shown by $M_{a}$, passive or resistive torque of pump is shown by $M_{p}$ and viscous torque is $M_{\zeta}$ [18]. Network frequency is shown by $f$ and it is assumed that stator pole pair number is one. Here following equation shows the torque of the asynchronous motor.

$$
M_{M T}=k_{M T} U^{2}(2 \pi f-\omega)
$$

Viscous torque and passive torque can be represented by 


$$
\begin{array}{r}
M_{\zeta}=k_{\zeta} \omega \\
M_{p}=\frac{\rho g Q_{v 2} H}{\eta_{p} \omega}
\end{array}
$$

Equation 18 shows the basic parameters of the centrifugal pump and pump flow rate is shown by $Q, H$ shows the pump head and the angular velocity is shown by $\omega$. Peripheral cross section of the impeller channels and meridian component of velocity express the pump flow. Head value is proportional to angular velocity as flow rate is proportional to angular velocity [19].

In the last equation the pump efficiency coefficient is denoted by $\eta_{p}$ which is constant and in different modes it changes to some extent and this reflects to the other parameters.

The total operating system $H_{\text {Total }}$ can be defined as

$$
H_{\text {Total }}=H_{S}+H_{D}+\left(P_{R T}-P_{R E S}\right)
$$

Here static head is represented by $H_{S}$, dynamic head is shown by $H_{D}$, pressure in the surface of the water in the receiving tank is shown by $P_{R T}$ and the pressure in the surface of the water in the reservoir tank is represented by $P_{R E S}$ [20].

Based on pump height pressure changes and it is considered negligible value.. But atmospheric pressure changes with the height. Equation shows the change in pressure and elevation difference between the reservoir and receiving tank. But this is not so significant and considered as negligible.

$$
P_{R T}-P_{R E S} \approx 0
$$

So the equation will be

$$
H_{\text {Total }}=H_{S}+H_{D}
$$

The difference between the point of discharge and the surface of the reservoir into the receiving tank is static head which is shown by $H_{s}$. Between maximum and minimum head value, static head for the system will vary because water level of the reservoir also varies.

$$
\begin{gathered}
H_{S_{\min }}=\text { discharge level }- \text { reservoir } T W L \\
H_{S_{\max }}=\text { discharge level }- \text { reservoir } B W L
\end{gathered}
$$

Here top water level is TWL and bottom water level is BWL.

Within the system as a result of friction dynamic head is generated. Basic Darcy Weisbach equation helps to calculate the dynamic head

$$
H_{D}=\frac{K v^{2}}{2 g}
$$

Here the loss coefficient is shown by $K$, velocity in the pipe is shown by $v$ and acceleration is $g$.

Now velocity is shown as

$$
v=\frac{Q}{A}
$$

Here flow rate is shown by $Q$ through the pipe and cross sectional area is shown by $A$.

Area $A$ is shown as 


$$
A=\frac{\pi D^{2}}{4}
$$

The loss coefficient $K$ is form of two elements:

$$
K=K_{\text {fittings }}+K_{\text {pipe }}
$$

$K_{\text {fittings }}$ is shown as pumping the water from reservoir to receiver tank fittings used for the pipeworks of the system $K_{\text {pipe }}$ is associated with length of pipe ,friction and the diameter of the pipe.

$$
K_{\text {pipe }}=\frac{f L}{D}
$$

Here $f$ shows the friction factor, $L$ shows the pipe length and $D$ is the pipe diameter. By modified version of Colebrook White equation the friction coefficient $f$ can be found.

$$
f=\frac{0.25}{\left[\log \left\{\frac{k}{3.7 \times D}+\frac{5.74}{R e^{0.9}}\right\}\right]^{2}}
$$

Here roughness factor is $k$ and Reynolds number is $R e$. The roughness factor $k$ is a standard fixed value collected from standard tables and it depends on the material of the pipe and pipe condition. For any flow in pipe, the following formula is used for the calculation of Reynolds number [21]:

$$
R e=\frac{v D}{\vartheta}
$$

$\vartheta$ is the kinematic viscosity. Operation of the pumping system is based on affinity law. First affinity law is shown in equation where flow $Q$ is proportional to shaft speed $N$.

$$
\frac{Q_{1}}{Q_{2}}=\frac{N_{1}}{N_{2}}
$$

As per, second affinity law, head is proportional to the square of the shaft speed.

$$
\frac{H_{1}}{H_{2}}=\frac{\left(N_{1}\right)^{2}}{\left(N_{2}\right)^{2}}
$$

The power of the pump can be calculated as

$$
P=\frac{Q \times H \times g \times \rho}{\text { Pump Efficiency }}
$$

Here $P$ is the power requirement for pump, $H$ is the head, $g$ is the acceleration gravity, $\rho$ is the density of water.

\section{Experimental analysis}

The experiment has been done with 3 phase, $50 \mathrm{~Hz}, 415 \mathrm{~V}, 0.75 \mathrm{HP}$ squirrel cage induction motor coupled with VFD based centrifugal pump with 2800 RPM speed and 23.5 meter head value. At healthy conditions three phase induction motor produces only positive sequence currents and it is symmetrical system. When symmetry is disturbed during fault situation it generates positive, negative, and zero sequence. The experiment has been done by creating inter turn fault in induction motor and analyzing the parameter changes both for motor and coupled pump. A Simulink model of three phase induction motor with turn fault in one phase winding has been built with the help of MATLAB software. As experimentally it is challenging to create fault due to shorting of high percentage value, the Simulink model has been developed. After completing the developed model, the model is verified both in healthy and faulty conditions. In different levels 
of shorting in one phase winding the model is simulated and the phase current values are stored in MATLAB workspace. Negative sequence current, positive sequence current, and zero sequence currents are calculated from these values. The next step is to verify how the inter turn fault affects various parameters of pump which is coupled with induction motor. After the simulation process is over the results are verified by OP5700 realtime simulator (hardware in loop) for validation. In another part of experiment, ML algorithms have been implemented on simulation data collected through MATLAB for identification and prediction of fault in induction motor based pumping system and for analyzing which algorithm is suitable for detection of fault. The Simulink model has been built based on the mathematical equations given in section 3 . Figure 1 shows the block diagram of inter turn fault detection in induction motor based pumping system.

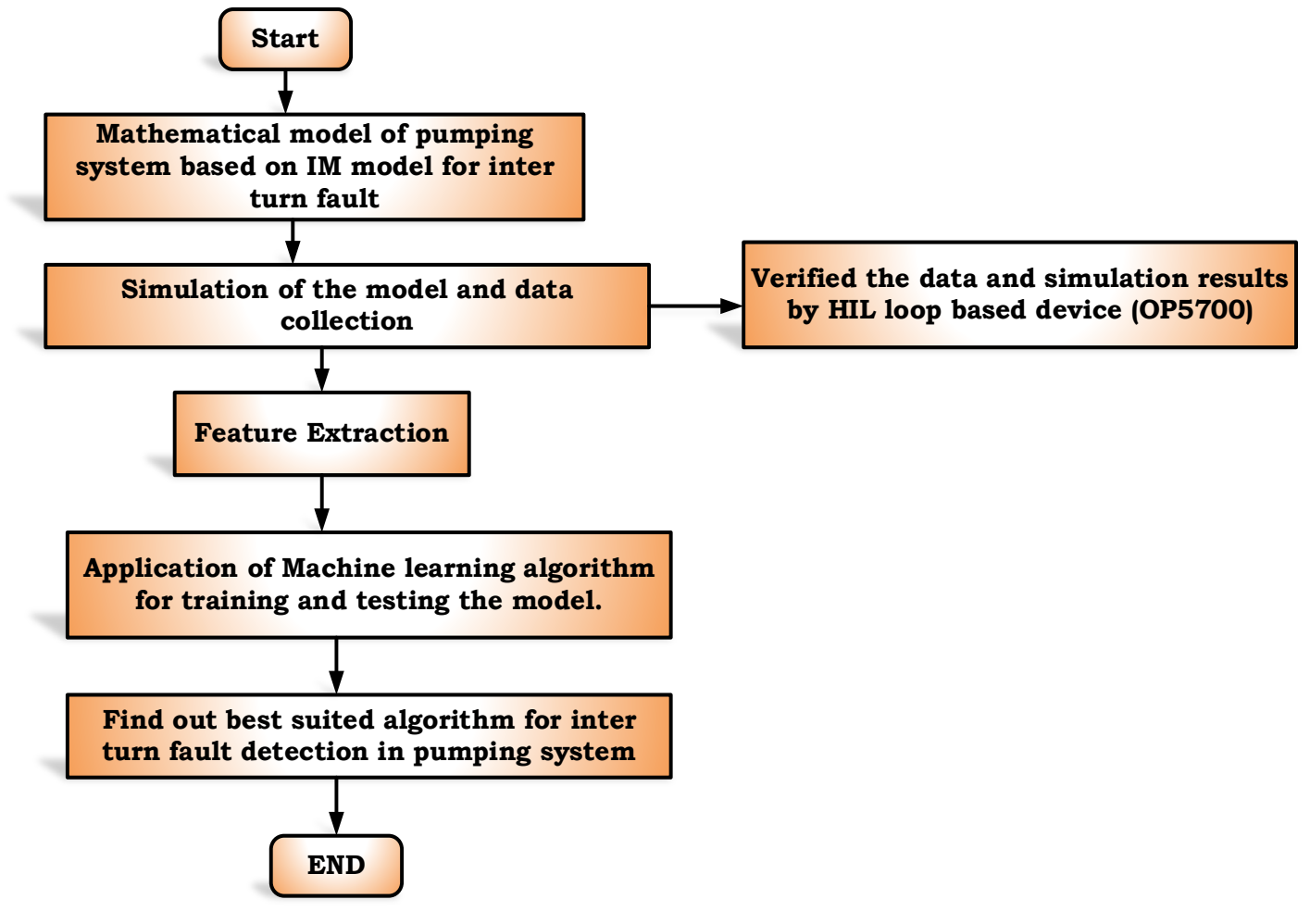

Figure 1. Flow chart of inter turn fault detection in induction motor based pumping system

The details of the induction motor are : stator resistance $R_{s}$ is $0.288 \Omega$, rotor resistance $R_{r}$ is $0.158 \Omega$, stator inductance $L_{s}$ and rotor inductance $L_{r}$ are $0.0425 \mathrm{H}$ and $0.0438 \mathrm{H}$ respectively, mutual inductance $L_{m}$ is $0.0412 \mathrm{H}$ and inertia $J$ is 0.4 . Here number of poles are 2 .

Main input parameter shows per unit changes from positive sequence current and to negative sequence current for classification of severity of the fault level in phase windings.

$$
\begin{gathered}
\delta=\frac{\text { (Positive Sequence Current }- \text { Negetive Sequence Current) }}{\text { Positive Sequence Current }} \\
\text { or } \delta=\frac{\left(I_{p}-I_{N}\right)}{I_{p}}
\end{gathered}
$$

When the system is in healthy condition there will be no short circuit turn. But when the system is in fault condition the negative sequence current will increase once the turn fault percentage will increase. In the proposed research upto $40 \%$ inter turn fault has been measured. $40 \%$, the value of $\delta$ varies from 1 to 0.98 , for short circuit level of $0 \%$ to $40 \%$ Figure 2 shows the Simulink model of induction motor based centrifugal pump system which has three phase source, VFD drives and induction motor coupled with pump. Table 1 shows the magnitude of phase current and sequence component current. 


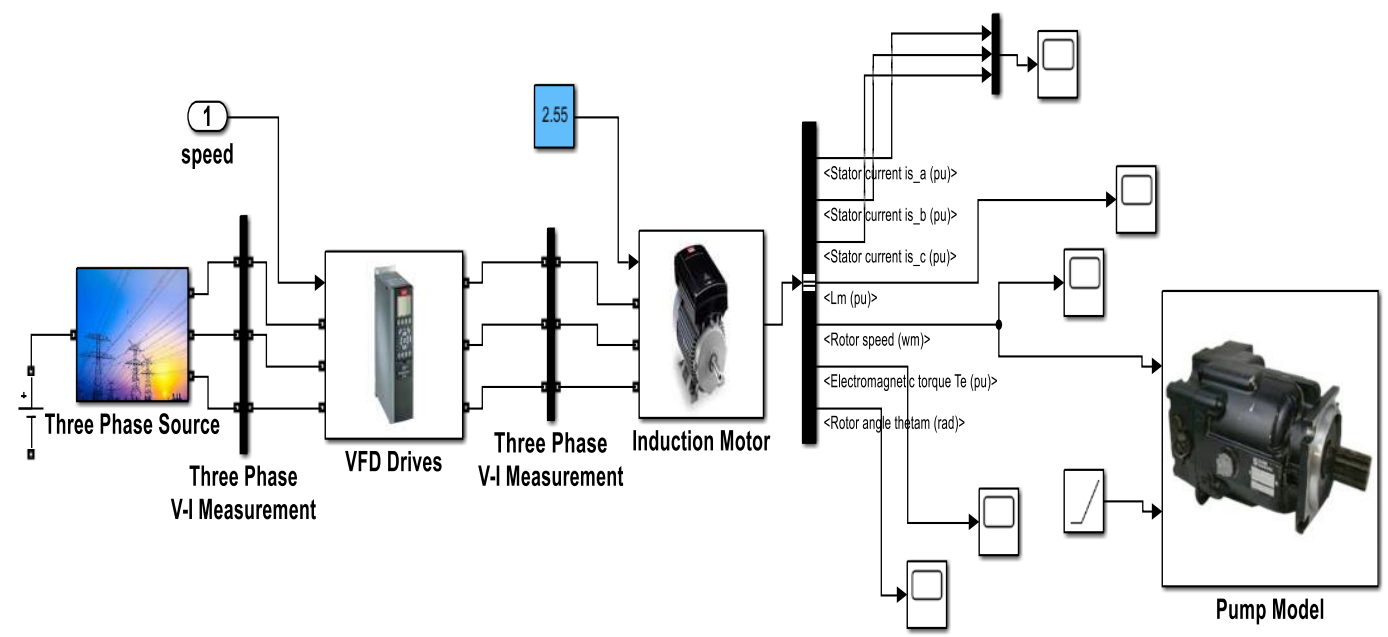

Figure 2: Simulink model of induction motor based centrifugal pump

Table 1: Magnitude of phase current and sequence component current in different \% shortings of Phase A winding

\begin{tabular}{|c|c|c|c|c|c|c|}
\hline \multirow{2}{*}{$\begin{array}{c}\% \text { Shorting } \\
\text { in phase A } \\
\text { windings }\end{array}$} & \multicolumn{3}{|c|}{ Phase Current value (A) } & \multicolumn{3}{|c|}{$\begin{array}{c}\text { Sequence components of current } \\
\text { values }\end{array}$} \\
\hline & $I_{a}$ & $I_{b}$ & $I_{c}$ & $I_{\text {positive }}$ & $I_{\text {negetive }}$ & Izero \\
\hline 0 & 1.54 & 1.52 & 1.53 & 1.601 & 0.0042 & 0.0042 \\
\hline 1.5 & 1.62 & 1.59 & 1.60 & 1.628 & 0.0234 & 0.02312 \\
\hline 2 & 1.628 & 1.59 & 1.60 & 1.635 & 0.0238 & 0.0235 \\
\hline 5 & 1.64 & 1.59 & 1.61 & 1.646 & 0.0362 & 0.0369 \\
\hline 10 & 1.641 & 1.59 & 1.61 & 1.679 & 0.0451 & 0.0457 \\
\hline 15 & 1.66 & 1.60 & 1.615 & 1.681 & 0.0628 & 0.0630 \\
\hline 20 & 1.67 & 1.60 & 1.62 & 1.684 & 0.0653 & 0.0654 \\
\hline 25 & 1.68 & 1.605 & 1.621 & 1.691 & 0.0658 & 0.0661 \\
\hline 30 & 1.74 & 1.65 & 1.63 & 1.695 & 0.0671 & 0.0669 \\
\hline 35 & 1.82 & 1.68 & 1.69 & 1.699 & 0.0680 & 0.0670 \\
\hline 40 & 1.85 & 1.70 & 1.71 & 1.7 & 0.0683 & 0.0675 \\
\hline
\end{tabular}

Though the simulation model has been analysed from $0 \%$ to $40 \%$ short circuit fault, the HIL OP5700 results have been compared between healthy and $40 \%$ short circuit fault for checking the highest fluctuation during extreme fault condition. When inter turn fault occurs phase A current increases and it helps to increase the phase $B$ and phase $C$ current. During fault condition torque response of the motor suffers from oscillations. As torque is proportional to speed, if during fault condition torque increases, speed of the motor also increases. The motor is coupled with pump, so that speed is fed to pump also and once pump operates in fault condition, the flow rate value suddenly increases and pressure decreases. Now if the pressure goes below vapour pressure, cavitation problem will occur and sudden increase of flow rate creates vibrational problem in the overall system.

\section{Results and Discussion}

The figures show the performance curve of current, speed, torque with respect to time and pump curves for flowrate vs head value both in healthy and faulty condition. All the results are obtained from OP5700 HIL based device which verified the simulation results. Figure 3 (a) and 3 (b) show the healthy and $40 \%$ inter turn fault condition of stator current. Phase A, B and C current increases as fault occurs. Figure 4 (a) and figure 4 (b) show the healthy and $40 \%$ inter turn fault condition speed value. Similarly Figure 5(a) and figure 5(b) show the healthy and $40 \%$ inter turn fault condition torque value. During fault condition motor suffers from oscillations. The size of the oscillations changes when the percentage of turn increases at same load condition. As the oscillations lead to increase in the rated power of the machine, the oscillation in the torque also increases. 


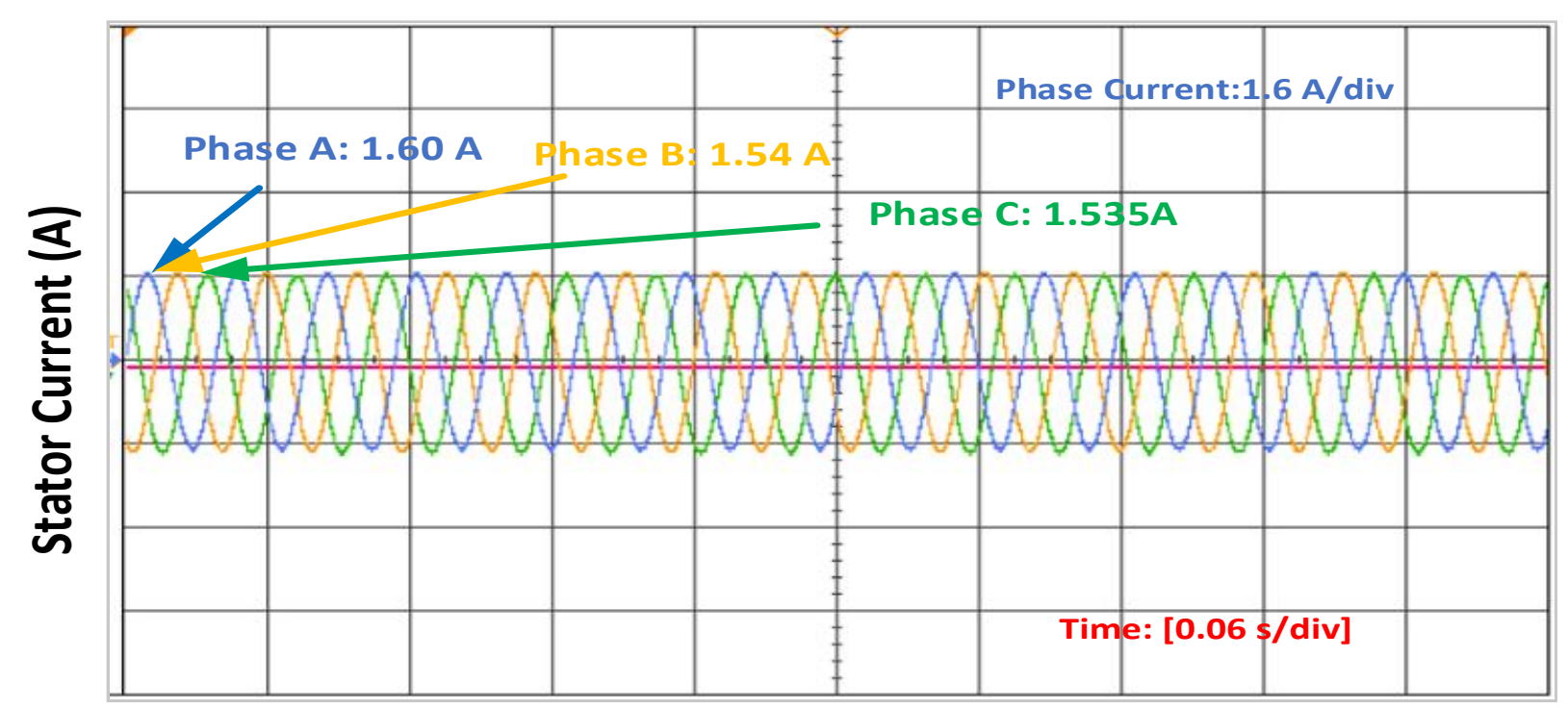

(a)

Time (S)

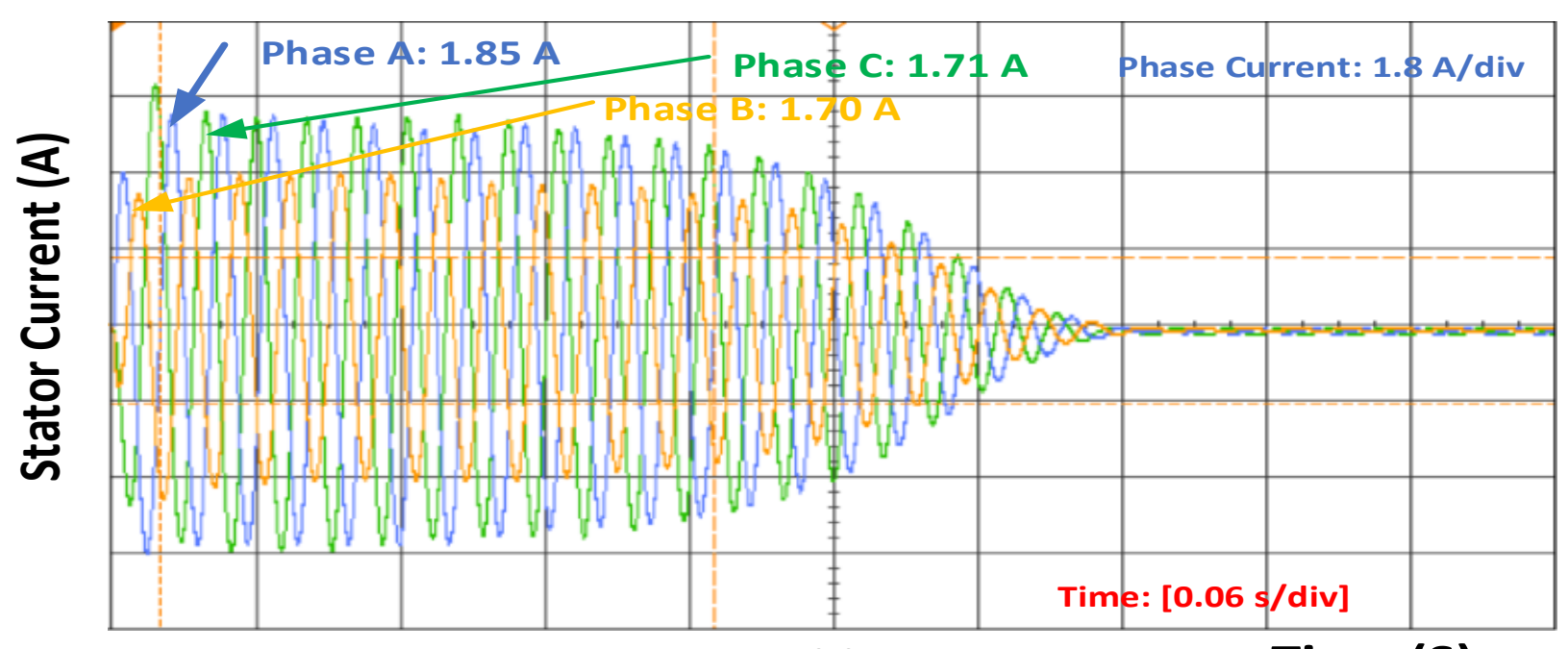

(b)

Time (S)

Figure 3 (a) Stator current in healthy condition for induction motor, Figure 3 (b) Stator current after $40 \%$ inter turn fault in induction motor. 


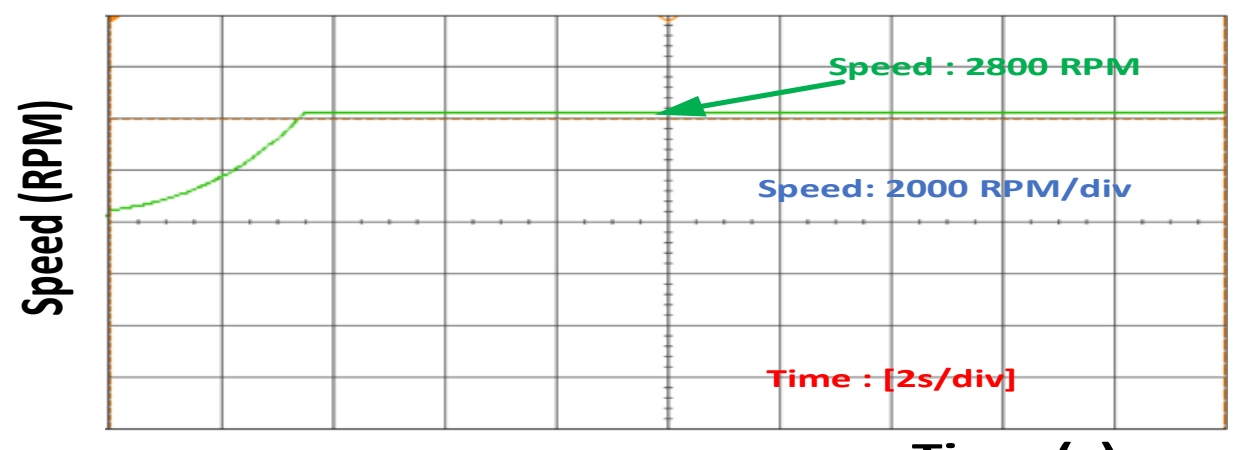

(a)

\section{Time (s)}

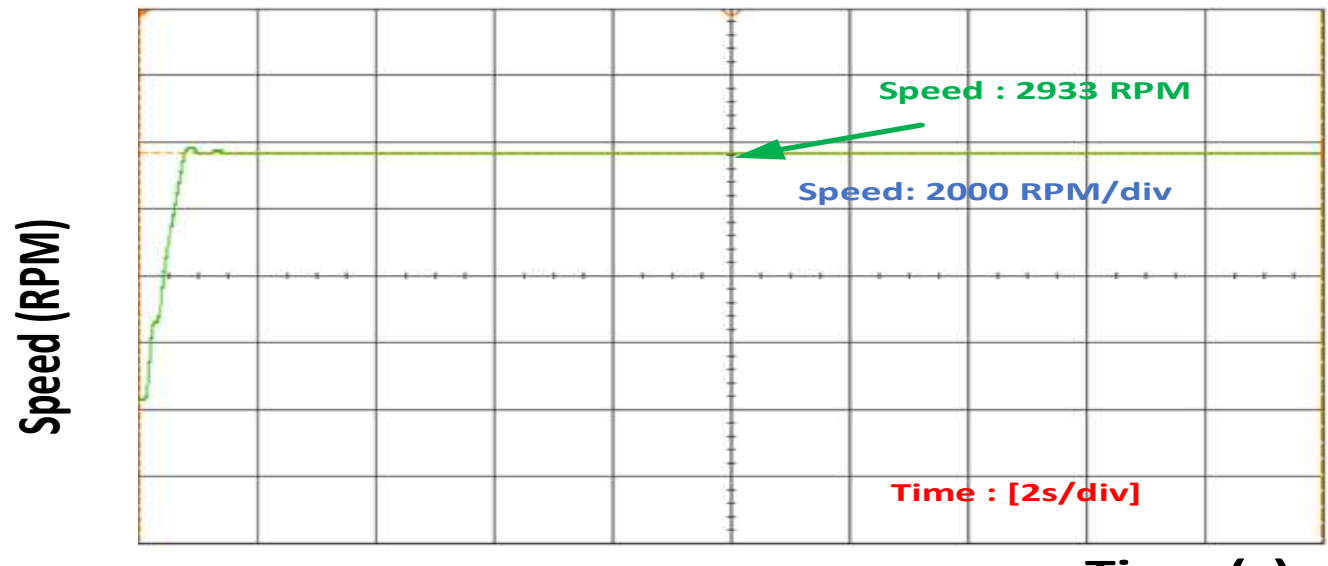

(b)

Time (s)

Figure 4 (a): Speed for induction motor in healthy condition. Figure 4 (b): Speed for induction motor $40 \%$ stator inter turn fault occurs.
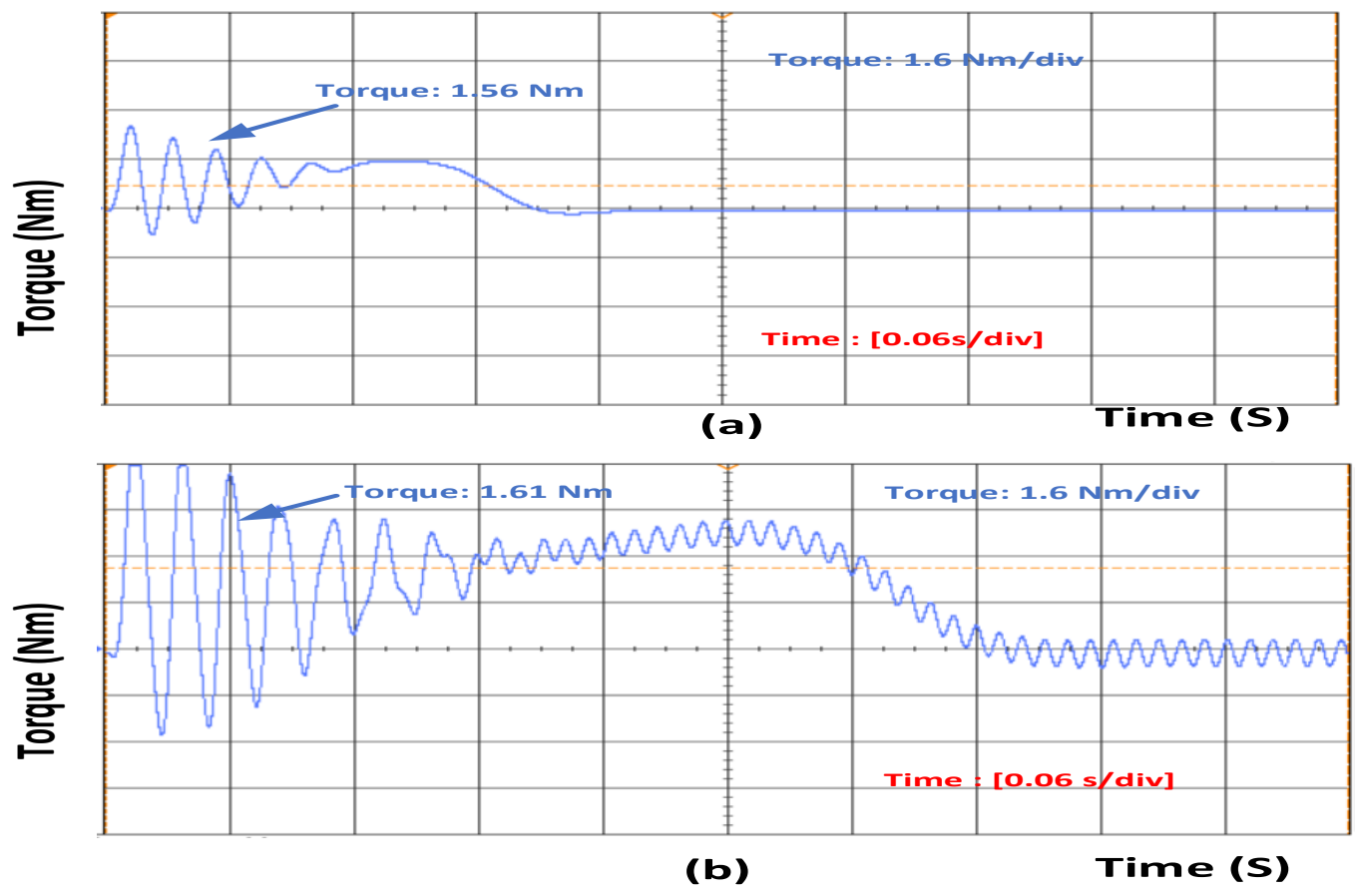

Figure 5 (a) Torque of the induction motor in healthy condition Figure 5 (b): Torque of the induction motor in faulty condition 
Figure 3 shows that current value increases for phase A. Once inter turn fault occurs and if the number of turn increases current value also increases. Increase in phase B and phase C current are accelerated also. Similarly speed and torque value also increase during fault condition as shown in figures 4 and 5 . Once the motor speed increases pump speed also increases. . Increase in speed causes increase in flow rate and decrease in head value. Figure 6 (a) and 6 (b) show the pump performance curve and system curve in healthy and $40 \%$ inter turn fault condition. Figure 7 shows the hardware setup of HIL device.

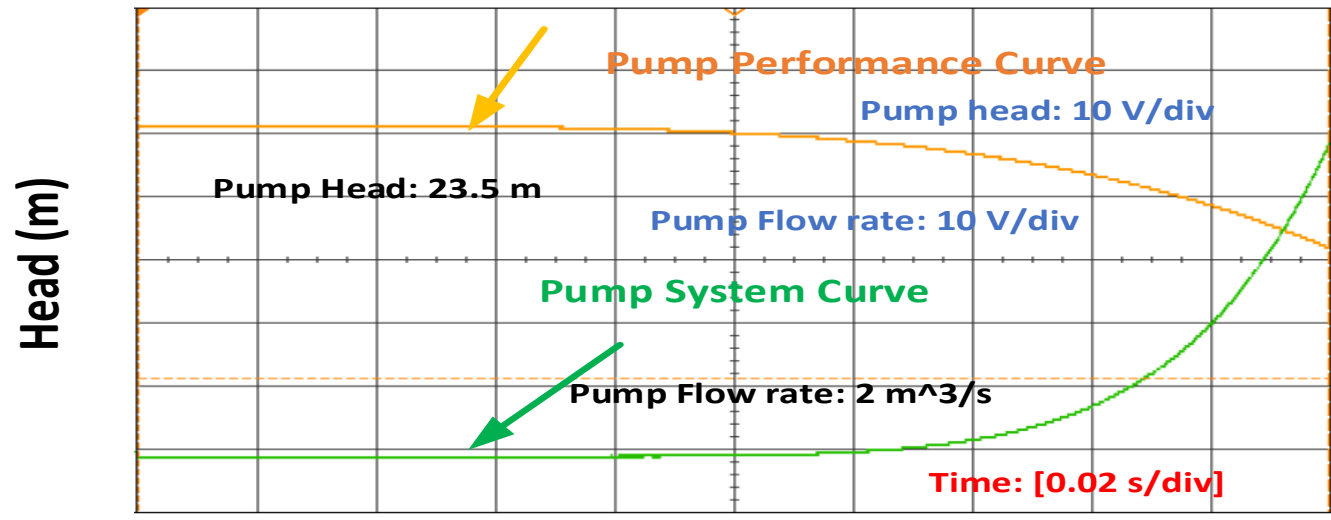

(a)

Flow rate $\left(\mathrm{m}^{\wedge} 3 / \mathrm{s}\right)$

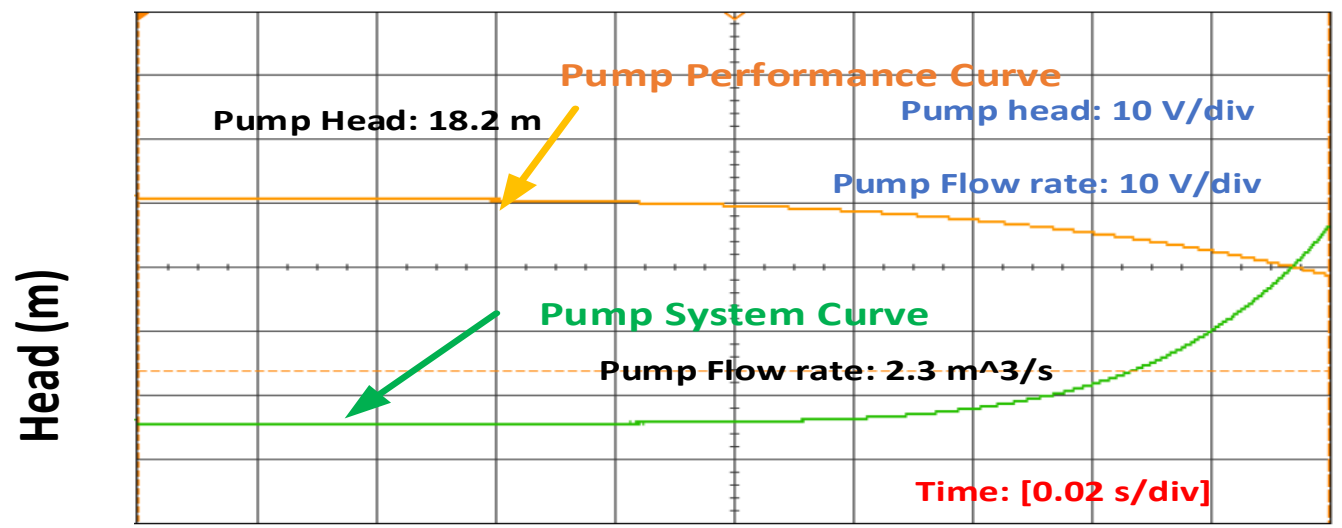

(b)

Flow rate $\left(m^{\wedge} 3 / s\right)$

Figure 6(a) Pump performance curve and system curve in healthy condition Figure 6(b) Pump performance curve and system curve in $\mathbf{4 0} \%$ inter turn fault condition.

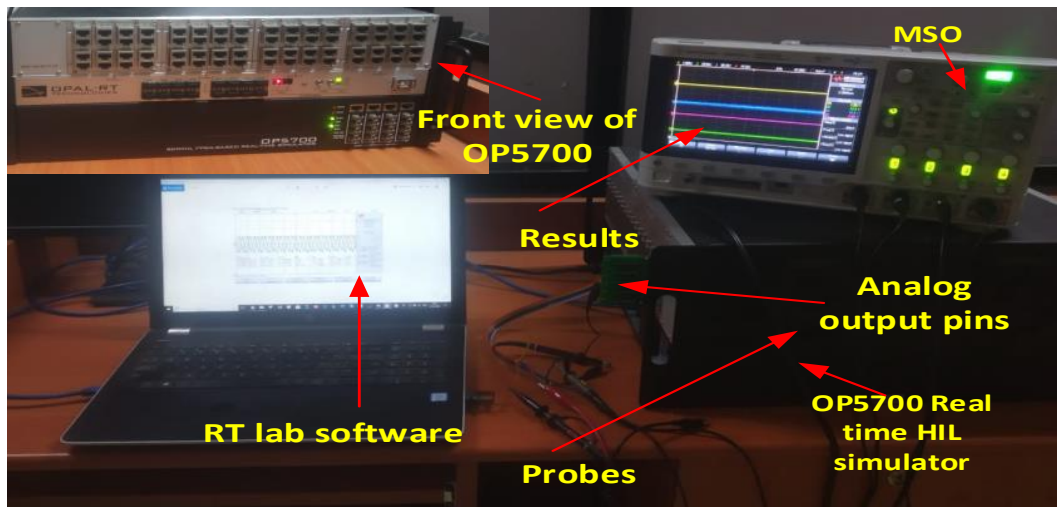

Figure 7: Hardware setup of HIL device 


\section{Application of ML approach in the induction motor based pump fault detection}

Generally $\mathrm{ML}$ algorithms are of two types, supervised and unsupervised, and supervised algorithms have target variables which are formed from the predicted value of input variables. Figure 8 shows the generalized block diagram of proposed research after the data collection for finding out the best suited algorithm.

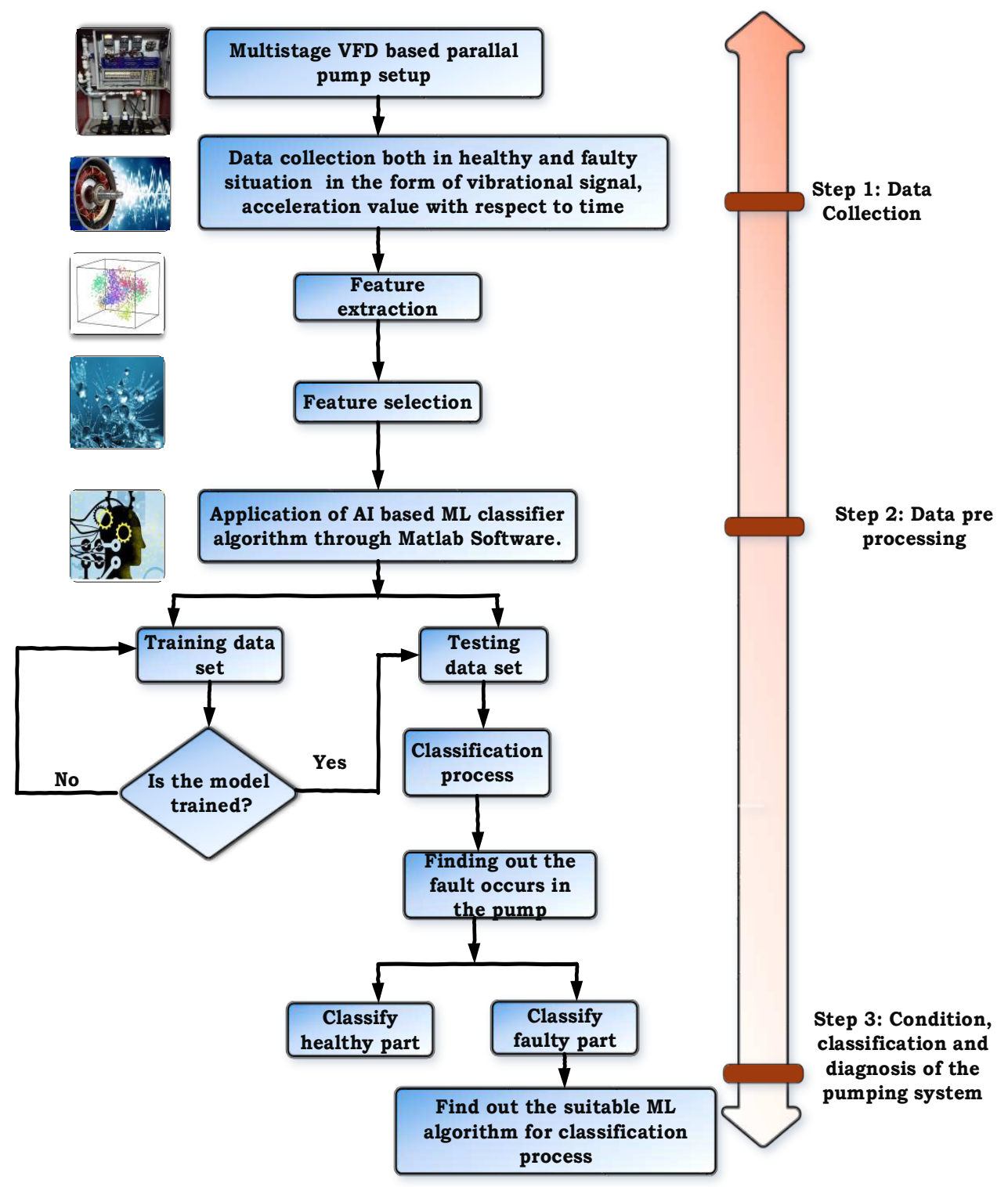

Figure 8: Block diagram of the proposed research for choosing best suited algorithm

The powerful technique ANN is used for the diagnosis of induction motor more accurately. Neural network (NN) is one of the pattern classifiers. Many problems can be solved by using pattern classification of NN $k$ and these problems involve in variable recognition and for induction motor fault diagnosis it cannot be entirely described or predicted. Mathematical model based computational algorithm is ANN which behaves like human brain and thinking process. It has various features like similar parallel processing, self-organizing, self-learning, classification and non-linear mapping abilities. Combination of Fuzzy and ANN is ANFIS [22] [23], and it is combined to improve speed, fault tolerance, adaptiveness and to obtain the better modelling system. Based on RMSE, $R^{2}$ value it can be compared which algorithm is suitable for inter turn fault detection in induction motor based pumping system.

For the inter turn fault detection in induction motor ANN and ANFIS models are proposed. Artificial immune system for ANN has self-adaptive control and performs better for continuous nonlinear function. The process can be done through online monitoring [24]. ANN is highly interconnected and the similarity is seen with human brain and it follows learning process like human being [25]. Units have interconnections between them and they have weights which are multiplied with the values which go through them. Unit has a fixed input which is known as bias and each unit form a weighted sum where bias is added. Transfer function analyzes this sum. Prediction of NN depends on training and testing data. The success of training is greatly 
affected by proper selection of inputs [26]. Learning process uses testing data and NN constructs input-output mapping. Iteration based on minimization or optimization of some error measured between the output produced and the desired output can be done adjusting the weights and bias. This process is repeated till an acceptable criterion for convergence is obtained. NN consists of input layer, hidden layer and output layer which is shown in figure 9. Output layer consists of six neurons like healthy condition, 5 turn short circuit, 10 turn short circuit, 20 turn short circuit, 30 turn short circuit and 40 turn short circuit. The algorithm can choose number of hidden layer by trial and error process. Levenberg Marquardt back propagation is chosen for the training purpose and training and testing data help to obtain average minimum square error (MSE) for ANN. For training purpose training rate is used for $70 \%$, for cross validation $15 \%$ and for testing $15 \%$. The average MSE values with respect to processing elements present in hidden layer are shown in table 2. With respect to processing elements percentage accuracy of hidden layers are shown for healthy and different turn fault condition in table2 and figure 10. Most of the cases the table and figure show that percentage of accuracy is 100 for healthy and faulty condition and average MSE is minimum $6.98 \mathrm{e}^{-8}$.

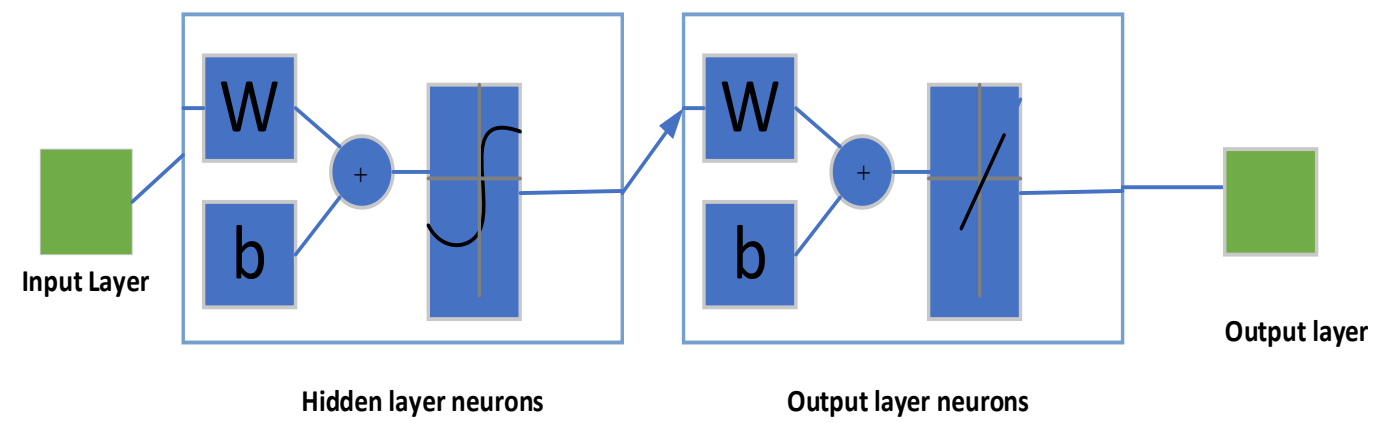

Figure 9: Block diagram of neural network

Table 2: Percentage accuracy of classification based on number of processing elements and MSE for ANN

\begin{tabular}{|c|c|c|c|c|c|c|c|}
\hline \multirow{2}{*}{$\begin{array}{c}\text { No of } \\
\text { Processing } \\
\text { Elements }\end{array}$} & \multirow[t]{2}{*}{ MSE } & \multicolumn{6}{|c|}{ Percentage accuracy of classification } \\
\hline & & $\begin{array}{l}\text { Healthy } \\
\text { condition }\end{array}$ & $\begin{array}{l}5 \text { turn } \\
\text { short } \\
\text { circuit }\end{array}$ & $\begin{array}{l}10 \text { turn } \\
\text { short } \\
\text { circuit }\end{array}$ & $\begin{array}{l}20 \text { turn } \\
\text { short } \\
\text { circuit }\end{array}$ & $\begin{array}{c}30 \text { turn } \\
\text { short } \\
\text { circuit }\end{array}$ & $\begin{array}{l}40 \text { turn } \\
\text { short } \\
\text { circuit }\end{array}$ \\
\hline 1 & 0.691 & 100 & 43 & 39 & 46.2 & 45.8 & 46.7 \\
\hline 2 & 0.540 & 85 & 54 & 45 & 67.1 & 56.9 & 54.3 \\
\hline 3 & 0.439 & 76 & 78 & 72 & 49.8 & 69.34 & 67.5 \\
\hline 4 & 0.231 & 100 & 96 & 61 & 78.1 & 78.9 & 79.2 \\
\hline 5 & 0.195 & 93 & 100 & 74 & 99.8 & 80.9 & 89.3 \\
\hline 6 & 0.0062 & 100 & 100 & 80 & 100 & 90.5 & 90.4 \\
\hline 7 & $1.23 e^{-2}$ & 86 & 100 & 100 & 100 & 95.6 & 100 \\
\hline 8 & $3.67 e^{-2}$ & 99 & 98 & 100 & 100 & 100 & 100 \\
\hline 9 & $4.68 e^{-6}$ & 100 & 100 & 100 & 99.5 & 100 & 100 \\
\hline 10 & $5.78 e^{-7}$ & 100 & 100 & 99 & 100 & 100 & 100 \\
\hline 11 & $6.98 e^{-8}$ & 100 & 100 & 100 & 100 & 100 & 100 \\
\hline
\end{tabular}




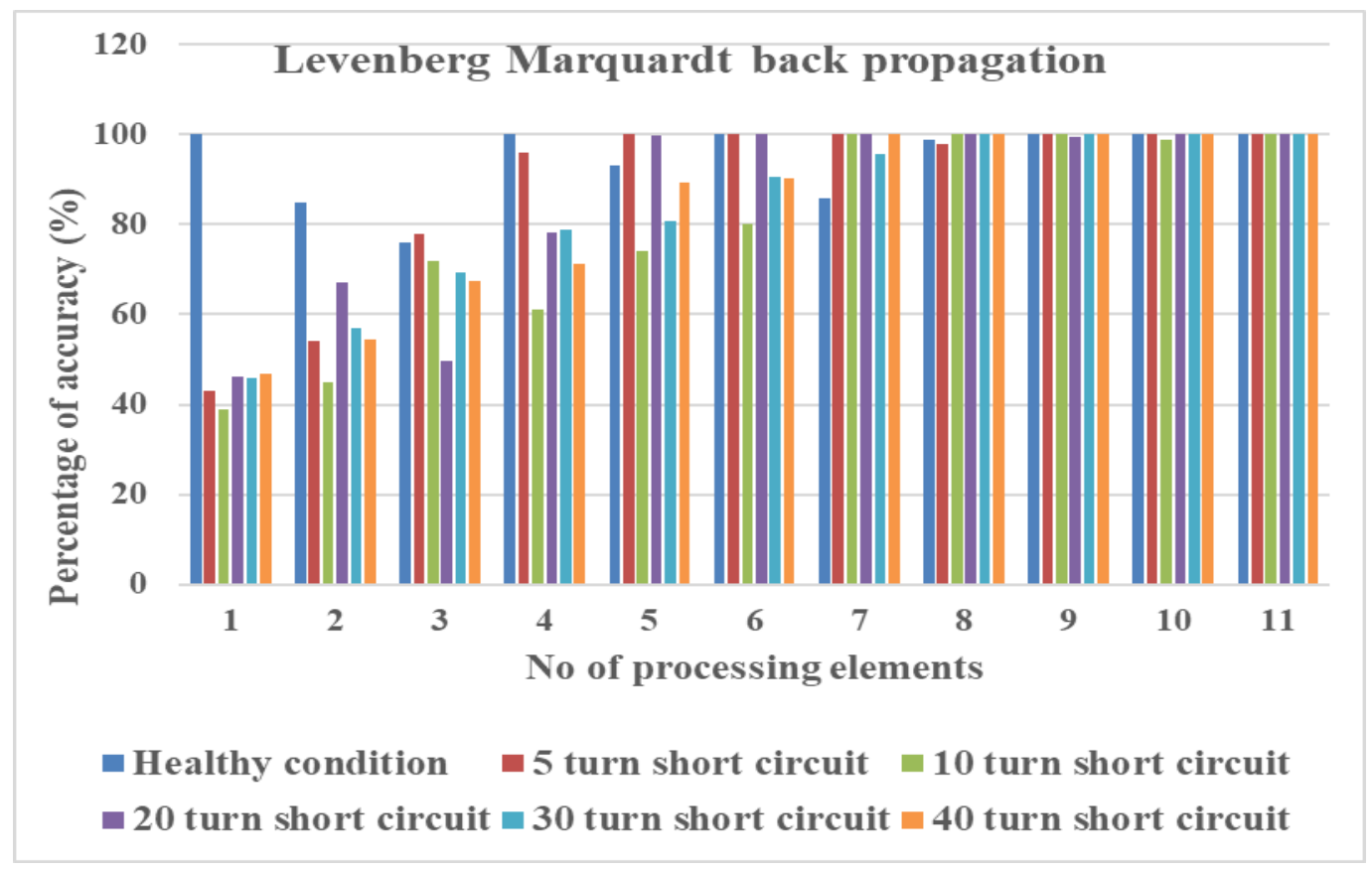

Figure 10: Percentage of accuracy with respect to processing elements in healthy and faulty condition

An intelligent system Neuro -Fuzzy technique ANFIS is used for modelling and control of ill-defined and uncertain systems. Input/output data pairs of the system under consideration builds ANFIS. ANFIS is the combination oOf ANN and Fuzzy which is used for the learning ability of fuzzy system. ANFIS consists of five layers [27] [28]. Layer 1 is fuzzification layer which calculates membership function. Layer 2 represents rules layer whose output is the firing strength of each node. Layer 3 highlights the normalization layer which normalizes the calculated firing strength. Layer 4 shows the consequent layer whose output layer is the product of normalized firing strength and the fuzzy rules consequent polynomial. Layer 5 shows the overall output and defines defuzzification layer whose output is overall ANFIS output. The problems of continuous changes in mobile learning environments are solved by ANFIS [29]. The proposed ANFIS model can be used for modelling the learner context. Defining input and output values, Fuzzy sets for input values, Fuzzy rules, and creating and training the NN are the steps applying ANFIS to learner model [30]. In the proposed work ANN model and ANFIS model both are implemented and compared to find out the better performance. $\mathrm{R}^{2}$ and RMSE are used to find out the best suited model for fault detection of induction motor based pumping system. The performances of the obtained ANN and ANFIS models are also compared after building the model is done. RMSE and $R^{2}$ have comparative statistical values for ANN and ANFIS models which are given in table 3. Validation data of the model is 0.05 . Prediction accuracy is also measured by $R^{2}$ and RMSE. Prediction accuracy for ANN ( $R^{2}$ is 100 and RMSE is 0.025$)$ is better than ANFIS model $\left(R^{2}\right.$ is 98.61 and RMSE is 0.08).

Table 3: Comparison of RMSE and $\mathbf{R}^{2}$ data for ANN and ANFIS

\begin{tabular}{|l|l|l|l|l|}
\hline Parameters & \multicolumn{2}{|l|}{ Training data } & \multicolumn{2}{l|}{ Testing data } \\
\cline { 2 - 5 } & ANN & ANFIS & ANN & ANFIS \\
\hline RMSE & 0.054 & 0.121 & 0.098 & 0.062 \\
\hline $\mathrm{R}^{2}$ & 0.998 & 0.934 & 0.969 & 0.897 \\
\hline
\end{tabular}


ANN and ANFIS models both performed well and are compatible for fault detection and able to predict the fault but based on RMSE and $\mathrm{R}^{2}$ of training and testing data, ANN performed better than ANFIS in the proposed experiment. The ANN model has been applied upto 200 epochs and best validation has been received in 150 epochs. Figure 11 shows the best fit value of the proposed model of ANN with respect to training, testing, validation and overall values.
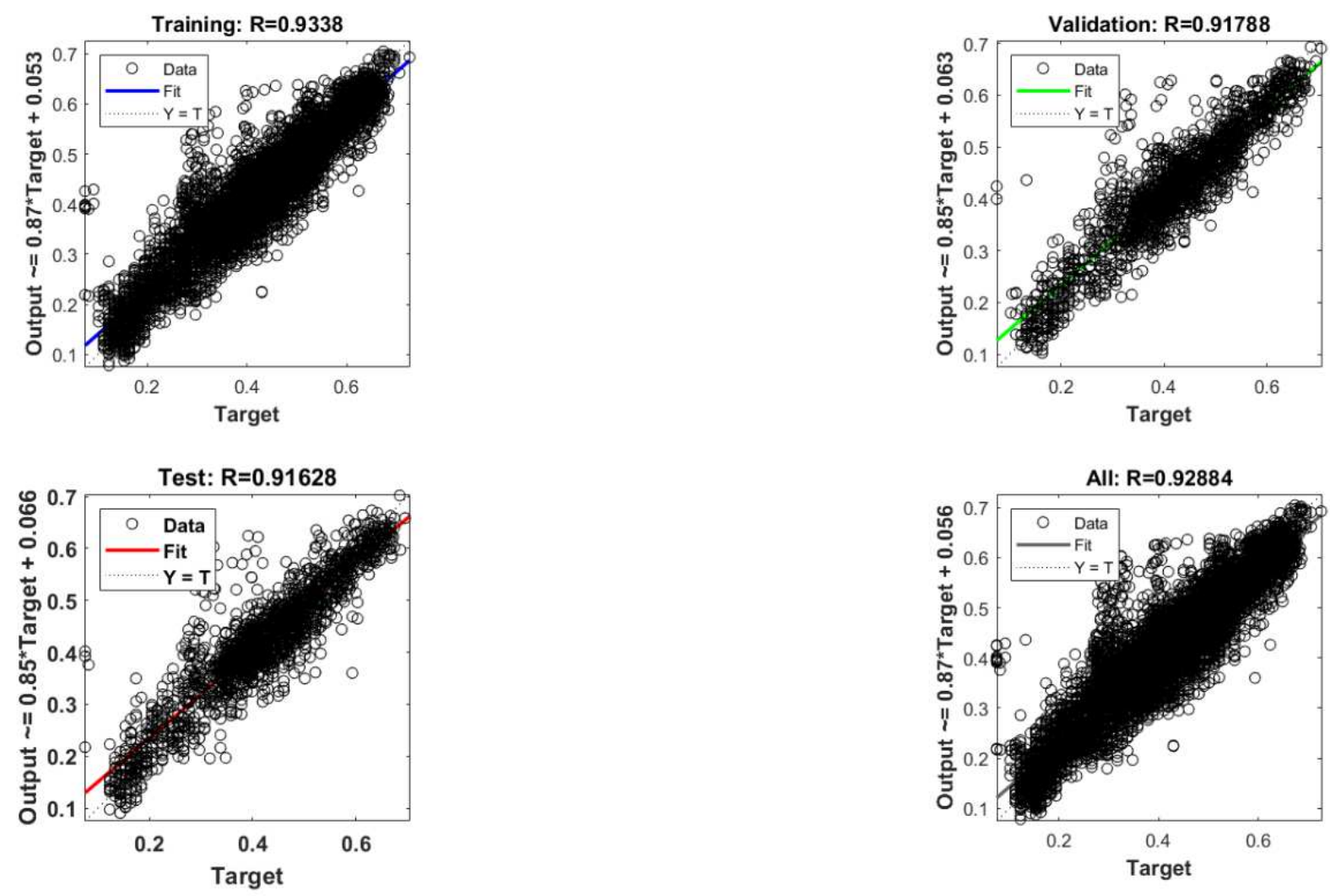

Figure 11: Best fit output of ANN model

Various previous works have been done for inter turn fault detection of induction motor and motor based pumping system. Till now researches on inter turn fault of induction motor analysed only changes of induction motor parameters after the fault occurs. But when the motor faces inter turn fault problem, the coupled pump is also affected and parameters of the pump also change. In the proposed research, including the change of parameters of inter turn fault affected motor, the change in pump parameters also have been analysed. Current coordinate transform algorithm for inter turn fault analysis in induction motor was developed. Mexbios development studio was built up to analyse the parameter changes in induction motor during fault condition. Though this is possible to implement in industrial applications, this process cannot predict the fault before the fault occurs and major damages are seen [31]. The proposed model is a simple easy process and helpful for less amount of data and predict the fault before massive failure. ANN algorithm for inter turn fault detection in induction motor with respect to various turns was used. Upto $10 \%$ inter turn fault was created in the experiment and phase A current changes were obtained. In the experiment per unit change of positive sequence current from negative sequence current was analysed. Experiment was done upto 54 epochs. Here experimental analysis was done for small level of values [32]. NN model in three different conditions which are no load, 50\% load and full load condition for five different motors for inter turn fault analysis was developed.. Upto 15\% inter turn fault was developed and the accuracy rate of NN model for various motors varied from $88 \%$ to $99 \%$ [33]. The novel wavelet analysis was developed in a research. Based on discrete wavelet transformation using Park's vector transformation the model was built for analysis of inter turn fault. Performance analysis was done for healthy and various turn fault conditions. MSE was obtained for performance accuracy analysis of healthy and faulty situation [34]. Other researches are based on FFT analysis and Park transformation but these researches are not suitable for predictive control model and not useful for heavy industrial application [35]. The proposed method of ANN has been used in current research which developed the Matlab model for analysis of the parameter changes of induction motor and pump during inter turn fault. As it is Simulink model so high range that is upto $40 \%$ of inter turn fault is possible to be 
created for analysis. Here ANN and ANFIS models have been implemented in the experimental results and it is found that performance of ANN is better than ANFIS model.

Similarly the authors also have implemented various supervised ML algorithms like SVM, K-NN, Decision tree, Naïve Bayes, Regression analysis with ANN and ANFIS. Based on accuracy rate, prediction speed and training time, algorithms are compared to find out most suitable algorithm for this experiment. Generally ML algorithms have target variables which are to be predicted from independent variables and these variables generate function for mapping of input to achieve desired output. After that training process should be done for the better achievement and for more accuracy. Until the desired accuracy rate is obtained the training process is going on. No target variable is required for unsupervised learning as it follows the clustering process. SVM is well known pattern recognition algorithm which is mainly used for classification and regression process. SVM has hyperplane and margin by which it separates the dataset and performs the classification task. Optimum hyperplane in the SVM maximizes the width of hyperplane to avoid the overlapping between the classes and this is the process of classification. Margins are classified between hard margin and soft margin. Since the present diagnosis deals with the non-linear classification problem, a soft margin is used. The accuracy of SVM depends on three factors like threshold function, cost function and kernel function. [34]. K-NN is non-parametric versatile learning algorithm which is also used for classification and regression problem. Instead of learning the discriminative function the algorithm memorizes the training dataset. By minimizing the training set intense based learning helps to avoid error. The disadvantages of K-NN are ample memory storage, long prediction time, and unnecessary sensitivity to irrelevant features. But when the data size is limited, K-NN works better than any other supervised learning algorithms. A decision tree dendritic classification model is used for both classification and regression problems. Breaking in smaller subset, classification process can be done and based on this feature selection can be done. The final structure is like tree branches and each node highlights the feature. Regression analysis provides user equation for graph for the prediction of the data. It always shows the weighted average value for the prediction purpose. Through the statistical analysis it can predict the accurate output. Most elementary statistical courses cover fundamental techniques, like making scatter plots and performing linear regression. Based on overall accuracy rate, prediction speed and training time the most suitable algorithm can be found out.

For experiment, features are divided into two categories randomly in the ratio of 70:30. In most of the cases $70 \%$ of data are used for training purposes and $30 \%$ are used for testing data for evaluation. For all the algorithms the rule is same. The entire diagnosis is carried through MATLAB pattern recognition app and classification learner app. Based on the evaluation, the accuracy rate of each algorithm is obtained using the formula. With the help of classification learner app the accuracy rate, prediction speed and training time of each algorithm have been analysed and compared.

$$
\text { Accuracy rate }(\%)=\frac{\text { Number of data diagnosed properly }}{\text { Total number of data diagnosed }} \times 100
$$

Table 4: performance analysis of various algorithms

\begin{tabular}{|llll|}
\hline Algorithms & $\begin{array}{l}\text { Accuracy } \\
\text { rate (percentage) }\end{array}$ & $\begin{array}{l}\text { Prediction Speed } \\
\text { (obs/sec) }\end{array}$ & $\begin{array}{l}\text { Training time } \\
(\mathrm{Sec})\end{array}$ \\
\hline SVM & 98.3 & 290 & 0.562 \\
\hline K-NN & 100 & 510 & 0.063 \\
\hline Naïve Bayes & 75.6 & 410 & 0.982 \\
\hline Decision Tree & 72.7 & 350 & 1.567 \\
\hline $\begin{array}{l}\text { Regression } \\
\text { Analysis }\end{array}$ & 90.9 & 467 & 2.987 \\
\hline ANN & & & 0.086 \\
\hline ANFIS & 99.6 & 480 & 4.236 \\
\hline
\end{tabular}

From the table 4 it is seen that performance of the K-NN and ANN are better for this research. But based on accuracy rate, prediction speed and training time K-NN is more suitable than ANN. Figure 12 picturizes the overall accuracy of the $\mathrm{ML}$ algorithms. 


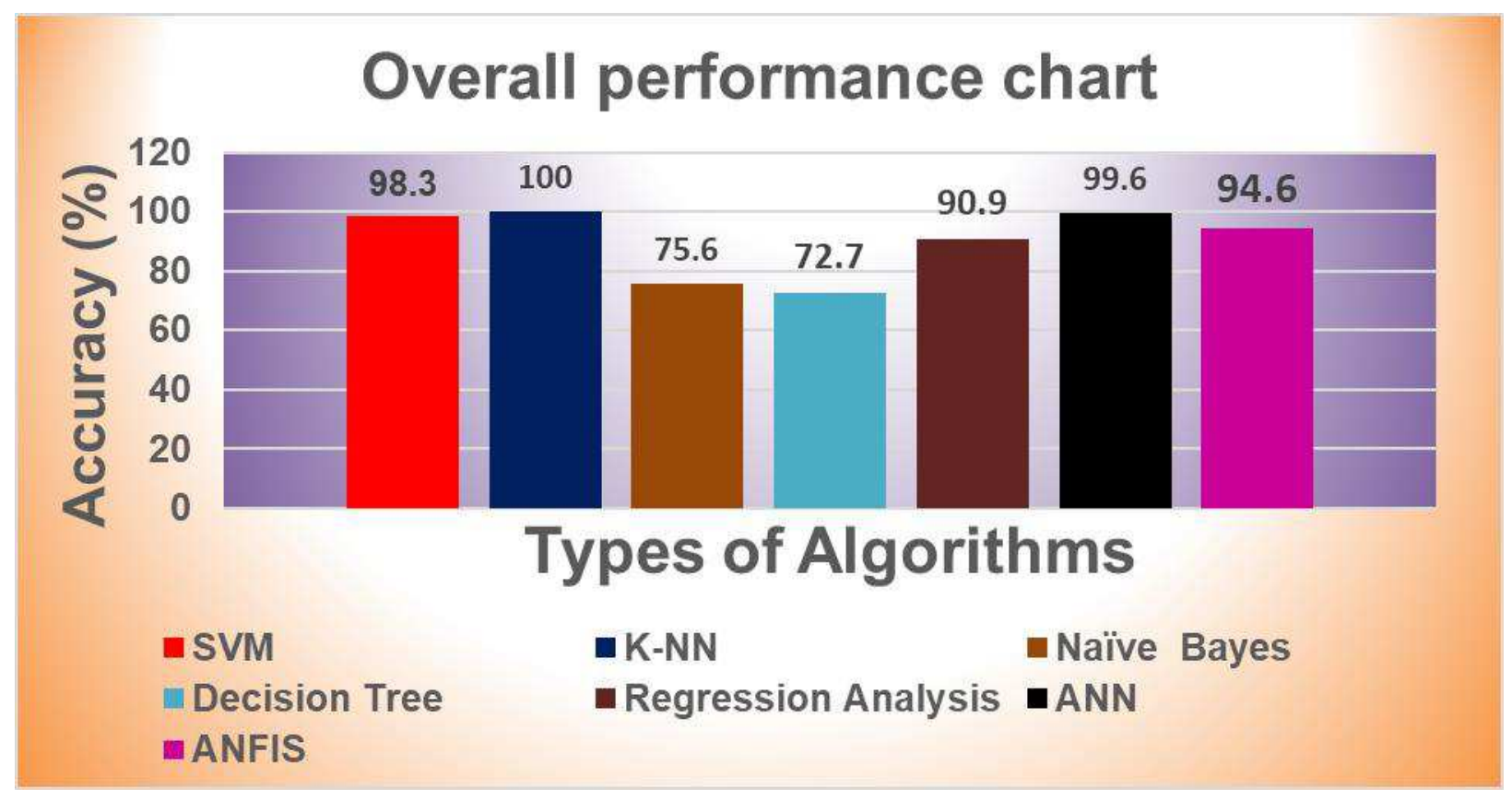

Figure 12; Overall accuracy of various ML algorithms

\section{Conclusion}

Inter turn fault analysis of induction motor based pumping system is explained in this article and the parameter changes during fault situation in different turn conditions have been shown. The simulation results have been verified through HIL loop based (OP5700) device and it is seen that phase current of motor increases when the fault occurs. Once current increases speed and torque also increases and it affects the pumping system. Speed helps to increase the flow rate of the pump suddenly and it causes huge pressure drop and decrease in head value. If pressure drops drastically, cavitation problem occurs and sudden increase in flow rate causes huge vibration in the pipe which causes water hammering problem. In this research at first by ANN and ANFIS algorithm based models identification and prediction of the fault have been done. Both the techniques are used and it is seen that ANN performs better than ANFIS, based on RMSE and $\mathrm{R}^{2}$ value. Various other research works are also compared with proposed work to find out the new development in the proposed work. It is observed that the proposed research is suitable for industrial application and can easily identify the faulty condition for large amount of data. In future the ANN would have been used for other fault detections in motor and pumping system and for other machineries also and can become a comprehensive diagnosis technique. The authors also compared various ML algorithms with ANN and ANFIS among which based on accuracy rate, prediction speed and training time it is seen that K-NN and ANN can work better for the proposed research. But based on overall accuracy rate K-NN works better than ANN. In addition, the deployment of the developed technique in a laboratory environment is an extension of the present work. More researches are possible through HIL based OP5700 device to verify the simulation results.

Author Contributions: Nabanita. Dutta, Palanisamy Kaliannan have done the inter turn fault analysis in induction motor based centrifugal pumping system. Expertise in the field of drives, control and fault analysis in pumping system using VFD and induction motor based pump has been shared by Paramasivam Shanmugam All authors have equally contributed to articulate the research work for its final depiction as a full research paper.

Funding: There is no source of funding for this research activity.

\section{Acknowledgment}

The authors like to express sincere gratitude to Danfoss Advanced Drives Laboratory, VIT University, for making this research work for execution and technical implementation in real-time. This is the industry-academic joint collaborative project for industrial pumping solution. The collaboration is between VIT Vellore and Danfoss Pvt Ltd industry.

Conflicts of Interest: The authors declare no conflict of interest. 


\section{References}

1. Neti, Prabhakar, and Subhasis Nandi. "Stator inter-turn fault analysis of reluctance synchronousmotor." In Canadian Conference on Electrical and Computer Engineering, 2005, pp. 1283-1286. IEEE, 2005.

2. Obeid, Najla Haje, Thierry Boileau, and Babak Nahid-Mobarakeh. "Modeling and diagnostic of incipient interturn faults for a three-phase permanent magnet synchronous motor." IEEE Transactions on Industry Applications 52, no. 5 (2016): 4426-4434.

3. Gao, Fengyang, Guoheng Zhang, Mingming Li, Yunbo Gao, and Shengxian Zhuang. "Inter-turn Fault Identification of Surface-Mounted Permanent Magnet Synchronous Motor Based on Inverter Harmonics." Energies 13, no. 4 (2020): 899.

4.Maraaba, Luqman S., Ssennoga Twaha, Azhar Memon, and Zakariya Al-Hamouz. "Recognition of Stator Winding Inter-Turn Fault in Interior-Mount LSPMSM Using Acoustic Signals." Symmetry 12, no. 8 (2020): 1370.

5.Yassa, Nacera, and M. Rachek. "Modeling and detecting the stator winding inter turn fault of permanent magnet synchronous motors using stator current signature analysis." Mathematics and Computers in Simulation 167 (2020): 325-339.

6.Kim, Byeong-Woo, Kyung-Tae Kim, and Jin Hur. "Simplified impedance modeling and analysis for inter-turn fault of IPM-type BLDC motor." Journal of Power Electronics 12, no. 1 (2012): 10-18.

7.Kalaskar, Chaitali S., and Vitthal J. Gond. "Motor current signature analysis to detect the fault in induction motor." International Journal of Engineering Research and Applications 4, no. 6 (2014): 58-61.

8.Mohanty, Amiya Ranjan, Prasanta Kumar Pradhan, Nitaigour P. Mahalik, and Sabyasachi G. Dastidar. "Fault detection in a centrifugal pump using vibration and motor current signature analysis." International Journal of Automation and Control 6, no. 3-4 (2012): 261-276.

9.Orrù, Pier Francesco, Andrea Zoccheddu, Lorenzo Sassu, Carmine Mattia, Riccardo Cozza, and Simone Arena. "Machine learning approach using MLP and SVM algorithms for the fault prediction of a centrifugal pump in the oil and gas industry." Sustainability 12, no. 11 (2020): 4776.

10. Sołjan, Zbigniew, Grzegorz Hołdyński, and Maciej Zajkowski. "CPC-Based Minimizing of Balancing Compensators in Four-Wire Nonsinusoidal Asymmetrical Systems." Energies 14, no. 7 (2021): 1815.

11. HS, Ranganatha Chakravarthy, Sai Charan Bharadwaj, S. Umashankar, Sanjeevikumar Padmanaban, Nabanita Dutta, and Jens Bo Holm-Nielsen. "Electrical fault detection using machine learning algorithm for centrifugal water pumps." In 2019 IEEE International Conference on Environment and Electrical Engineering and 2019 IEEE Industrial and Commercial Power Systems Europe (EEEIC/I\&CPS Europe), pp. 1-6. IEEE, 2019.

12. Dutta, Nabanita, Palanisamy Kaliannan, and Umashankar Subramaniam. "Effect of Motor Vibration Problems on Power Quality of Water Pumping at Residency." In IOP Conference Series: Materials Science and Engineering, vol. 937, no. 1, p. 012019. IOP Publishing, 2020. 
13. Arun Shankar, V. K., Umashankar Subramaniam, Sanjeevikumar Padmanaban, Jens Bo HolmNielsen, Frede Blaabjerg, and S. Paramasivam. "Experimental investigation of power signatures for cavitation and water hammer in an industrial parallel pumping system." Energies 12, no. 7 (2019): 1351.

14. Jain, Geet, and S. Umashankar. "Modelling and simulation of solar photovoltaic fed induction motor for water pumping application using perturb and observer MPPT algorithm." In 2016 International Conference on Energy Efficient Technologies for Sustainability (ICEETS), pp. 250254. IEEE, 2016.

15. Nabanita, D., S. Sreedhar, Neha Zade, Onkar Bhalerao, Pratik Potdar, and R. Raja Singh. "Inter turn Fault Analysis on Wound Rotor Induction Machine." In IOP Conference Series: Materials Science and 2019, Engineering, vol. 623, no. 1, p. 012016. IOP Publishing.

16. Hussein, HA Taha, M. E. Ammar, and MA Moustafa Hassan. "Three phase induction motor's stator turns fault analysis based on artificial intelligence." International Journal of System Dynamics Applications (IJSDA) 6, no. 3 (2017): 1-19.

17. Skowron, Maciej, Teresa Orlowska-Kowalska, Marcin Wolkiewicz, and Czeslaw T. Kowalski. "Convolutional neural network-based stator current data-driven incipient stator fault diagnosis of inverter-fed induction motor." Energies 13, no. 6 (2020): 1475.

18. Salih, Hamad Raad, Ali Abdulwahhab Abdulrazzaq, and Basarab Dan Guzun. "Dynamic Modeling of Pump Drive System utilizing Simulink/MATLAB Program." International Research Journal of Engineering and Technology (IRJET) 3, no. 1 (2016): 21-24.

19. Husari, Fatimatelbatoul, and Jeevanand Seshadrinath. "Incipient Inter Turn Fault Detection and Severity Evaluation in Electric Drive System Using Hybrid HCNN-SVM Based Model." IEEE Transactions on Industrial Informatics (2021).

20. Ostojic, Pero, Arijit Banerjee, Dhaval Patl, Wrichik Basu, and Shahid Ali. "Advanced motor monitoring and diagnostics." In Industry Applications Society 60th Annual Petroleum and Chemical Industry Conference, pp. 1-9. IEEE, 2013.

21. Haroun, S., A. Nait Seghir, and S. Touati. "Stator inter turn fault and voltage unbalance detection and discrimination approach for an reactor coolant pump." In 3rd International Conference on Systems and Control, pp. 99-104. IEEE, 2013.

22. Mohamed, Menshawy A., Mohamed A. Moustafa Hassan, Fahad Albalawi, Sherif SM Ghoneim, Ziad M. Ali, and Mostafa Dardeer. "Diagnostic Modelling for Induction Motor Faults via ANFIS Algorithm and DWT-Based Feature Extraction." Applied Sciences 11, no. 19 (2021): 9115.

23. Rajeswari, R., and N. Kamaraj. "Diagnosis of inter turn fault in the stator of synchronous generator using wavelet based ANFIS." World Academy of Science, Engineering and Technology 36 (2007): 203-209.

24. Panda, S., D. P. Mishra, and S. N. Dash. "Comparison of ANFIS and ANN Techniques in Fault Classification and Location in Long Transmission Lines." In 2018 International Conference on Recent Innovations in Electrical, Electronics \& Communication Engineering (ICRIEECE), pp. 11121117. IEEE, 2018. 
25. Esakimuthu Pandarakone, Shrinathan, Yukio Mizuno, and Hisahide Nakamura. "A comparative study between machine learning algorithm and artificial intelligence neural network in detecting minor bearing fault of induction motors." Energies 12, no. 11 (2019): 2105.

26. Duan, Fang, and Rastko Zivanovic. "A model for induction motor with stator faults." In 2012 22nd Australasian Universities Power Engineering Conference (AUPEC), pp. 1-5. IEEE, 2012.

27. Samanta, S., J. N. Bera, and G. Sarkar. "An Intelligent Adaptive Neuro Fuzzy based Fault Diagnosis System for Severity and Phase Detection of Induction Machine."

28. Ghafari, Shahab Hasanzadeh. A fault diagnosis system for rotary machinery supported by rolling element bearings. Waterloo, ON, Canada: University of Waterloo, 2007.

29. Solodkiy, Evgeniy, Dmitry Dadenkov, and Saveliy Salnikov. "Detection of stator inter-turn short circuit in three-phase induction motor using current coordinate transformation." In 2019 26th International Workshop on Electric Drives: Improvement in Efficiency of Electric Drives (IWED), pp. 1-4. IEEE, 2019.

30. Ghorbanzadeh, Omid, Thomas Blaschke, Jagannath Aryal, and Khalil Gholaminia. "A new GIS-based technique using an adaptive neuro-fuzzy inference system for land subsidence susceptibility mapping." Journal of Spatial Science 65, no. 3 (2020): 401-418.

31. Rajamany, Gayatridevi, Sekar Srinivasan, Krishnan Rajamany, and Ramesh K. Natarajan. "Induction motor stator interturn short circuit fault detection in accordance with line current sequence components using artificial neural network." Journal of Electrical and Computer Engineering 2019 (2019).

32. Maraaba, Luqman, Zakariya Al-Hamouz, and Mohammad Abido. "An efficient stator inter-turn fault diagnosis tool for induction motors." Energies 11, no. 3 (2018): 653.

33. Umashankar, S., P. Sanjeevikumar, and S. Paramasivam. "Adaptive neuro-fuzzy inference system (anfis) based direct torque control of pmsm driven centrifugal pump." (2017).

34. Babaa, Fatima, and Ouafae Bennis. "An accurate inter-turn short circuit faults model dedicated to induction motors." International Journal of Electrical and Computer Engineering 11, no. 1 (2021): 9.

35. Irfan, Muhammad, and Adam Glowacz. "Design of a novel electric diagnostic technique for fault analysis of centrifugal pumps." Applied Sciences 9, no. 23 (2019): 5093. 